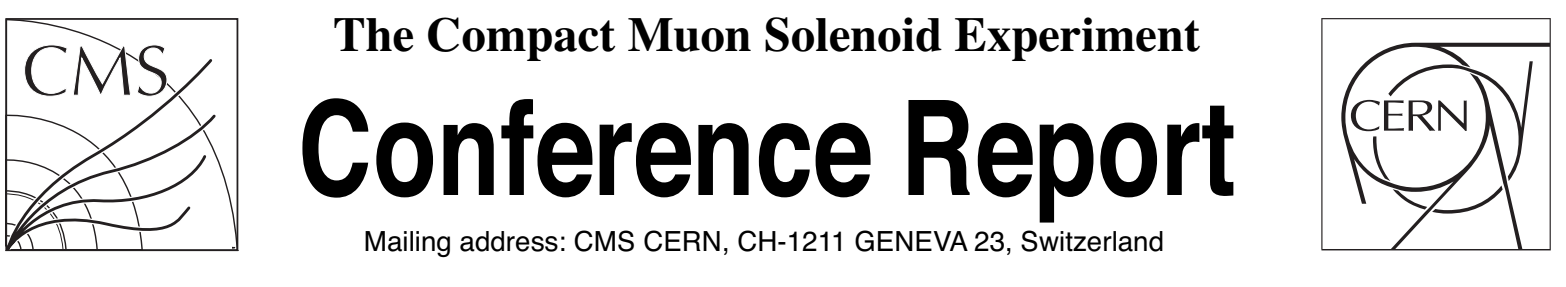

15 March 2019 (v3, 04 February 2020)

\title{
Development of the CMS MIP Timing Detector
}

\author{
Marco T. Lucchini \\ on behalf of the CMS Collaboration
}

\begin{abstract}
The Compact Muon Solenoid (CMS) detector at the CERN Large Hadron Collider (LHC) is undergoing an extensive Phase II upgrade program to prepare for the challenging conditions of the HighLuminosity LHC (HL-LHC). In particular, a new timing layer will measure minimum ionizing particles (MIPs) with a time resolution of $30 \mathrm{ps}$ and hermetic coverage up to a pseudo-rapidity of $|\eta|=3$. This MIP Timing Detector (MTD) will consist of a central barrel region based on L(Y)SO Ce crystals read out with SiPMs and two end-caps instrumented with radiation-tolerant Low Gain Avalanche Detectors. The precision time information from the MTD will reduce the effects of the high levels of pile-up expected at the HL-LHC and will bring new and unique capabilities to the CMS detector. The time information assigned to each track will enable the use of 4D reconstruction algorithms and will further discriminate interaction vertices within the same bunch crossing to recover the track purity of vertices in current LHC conditions. We present motivations for precision timing at the HL-LHC and the ongoing MTD R\&D targeting enhanced timing performance and radiation tolerance, including test beam results.
\end{abstract}




\title{
Development of the CMS MIP Timing Detector
}

\author{
Marco Lucchini ${ }^{\mathrm{a}}$, \\ on behalf of the CMS Collaboration \\ a Princeton University, Princeton, New Jersey
}

\begin{abstract}
The CMS detector at the CERN Large Hadron Collider is undergoing an extensive Phase II upgrade program to prepare for the challenging conditions of the High-Luminosity LHC. In particular, a new timing detector, the MTD, will measure minimum ionizing particles with a time resolution of $30-50 \mathrm{ps}$ and hermetic coverage up to a pseudo-rapidity of $|\eta|=3$. The MTD will consist of a central barrel region based on L(Y)SO:Ce crystals read out with SiPMs and two end-caps instrumented with radiation-tolerant low gain avalanche detectors. The precision time information from the MTD will reduce the effects of the high levels of pile-up expected at the High-Luminosity LHC and will bring new and unique capabilities to the CMS detector. The time information assigned to each track will enable the use of 4D reconstruction algorithms and will further discriminate interaction vertices within the same bunch crossing to recover the track purity of vertices in current LHC conditions. We present the motivations for precision timing at the High-Luminosity LHC, the overall detector design and technological choices including ongoing R\&D studies targeting enhanced timing performance and radiation tolerance.
\end{abstract}

Keywords: Precision Timing, HL-LHC detectors, Technology R\&D, crystals, SiPMs, Low Gain Avalanche Detectors

\section{Motivations for precision timing at HL-LHC}

The Compact Muon Solenoid (CMS) detector at the CERN Large Hadron Collider (LHC) will undergo a major upgrade to tackle the challenging conditions of the High-Luminosity LHC (HL-LHC) [1]. A particular challenge of such high luminosity phase will be the larger number of pileup interactions during collisions $(\sim 140--200)$ being a factor up to $\sim 5$ times higher than current pileup conditions. The probability density functions of the line density along the beam axis for the pileup of the LHC and for pileup 140 and 200 are shown in Fig. 1. For line densities above $1.2 \mathrm{~mm}^{-1}$, when the probability of spatial overlap of vertices increases, the time information provides an additional handle in vertex reconstruction. Under these conditions, the standard particle-flow algorithms used for event reconstruction begin to fail at a substantial rate. A typical distribution of vertices within a bunch crossing is shown in Fig. 2 featuring an RMS spread of about $4.5 \mathrm{~cm}$ in the spatial domain and about 180-200 ps in time. If one imagines slicing the beam spot into consecutive time exposures of 30-40 ps, the number of vertices per exposure drops down to the current LHC pileup levels of about 40. To address this challenge and tackle such high pileup environment, the CMS collaboration is thus planning to add a dedicated timing layer that will measure minimum ionizing particles (MIPs) with a time resolution of $30-40 \mathrm{ps,} \mathrm{referred} \mathrm{in} \mathrm{the}$ following as the MIP Timing Detector (MTD) [2].

Reduction of pile-up. As shown in Fig. 3, the use of time information reduces the number of tracks from pileup vertices that are incorrectly associated with a hard-interaction vertex. The addition of the MTD recovers a performance in pileup mitigation at 200 PU equivalent to the current LHC conditions and

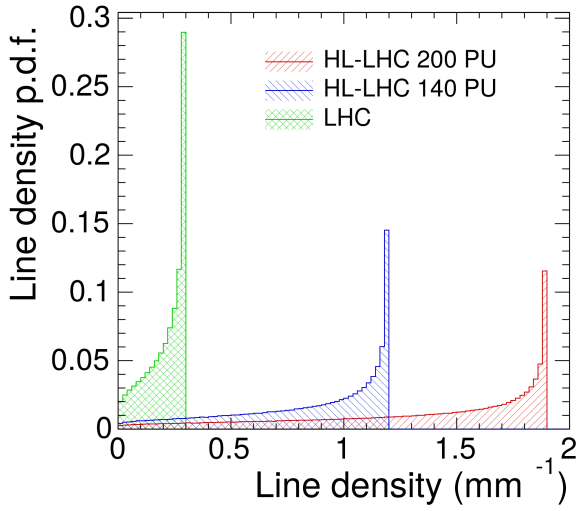

Figure 1: Probability density functions of the line density along the beam axis for the pileup of the LHC and for pileup 140 and 200.

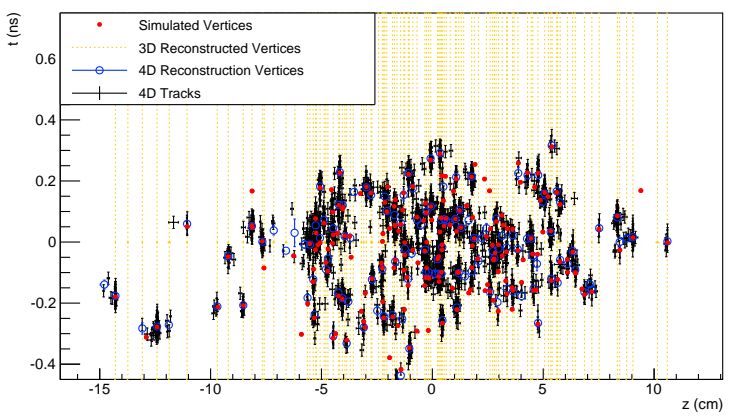

Figure 2: Simulated and reconstructed vertices in a 200 pileup event assuming a MIP timing detector covering the barrel and endcaps. The vertical yellow lines indicate 3D-reconstructed vertices, with instances of vertex merging visible throughout the event display. 


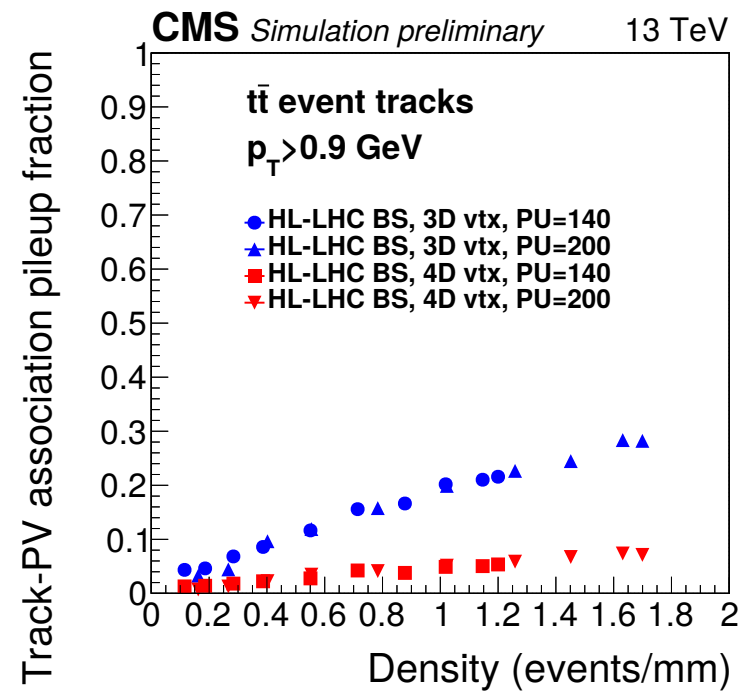

Figure 3: Number of pileup tracks incorrectly associated with the hard interaction vertex as a function of the collision line density for different time resolutions.

enhances the performance of b-jet tagging efficiency which relies on vertex reconstruction. By removing pileup tracks from the isolation cones, the new detector also improves reconstruction and isolation of photons and leptons as well as resolution of the missing transverse momentum. These improvements directly impact several key measurements of the HL-LHC physics program, for example the measurement of di-Higgs production.

Additional features enabled by precision timing. Besides preserving the quality of the data collected at the highest luminosities foreseen for HL-LHC, the MTD also enables new measurements and expand the potential of the CMS detector. An example is the MTD capability to identify charged hadrons as pions, kaons, or protons based on time-of-flight up to a few $\mathrm{GeV}$ in $p_{T}$, which is beneficial for the Heavy Ion physics and for specialized QCD studies in pp collisions. The MTD also represents a unique tool to extend the potential of searches for long-lived particles (LLPs) predicted by several BSM physics models. This is due to the MTD capability to reconstruct the time of displaced vertices by measuring the LLP's relativistic velocity parameter, $\beta$, and, in certain cases, permitting the reconstruction of the LLP's mass.

\section{Overview of the CMS MIP Timing Detector}

To optimally exploit the potential of precision timing, the MTD is designed as a nearly hermetic detector with coverage for a pseudo-rapidity range of $|\eta|<3$. The detector features a high efficiency for detection of charged particles with time resolution of 30-40 ps. To comply with the overall CMS structure, the MTD is divided in a barrel region, BTL, $(|\eta|<1.5$, surface $\left.\sim 38 \mathrm{~m}^{2}\right)$ and two endcaps, ETL, $(1.6<|\eta|<3$, surface $\sim 10 \mathrm{~m}^{2}$ ) as illustrated in Fig. 4 Since radiation levels in the inner rings of the endcaps, closer to the beam pipe, will be up to a factor 30 higher than in the barrel, different technologies

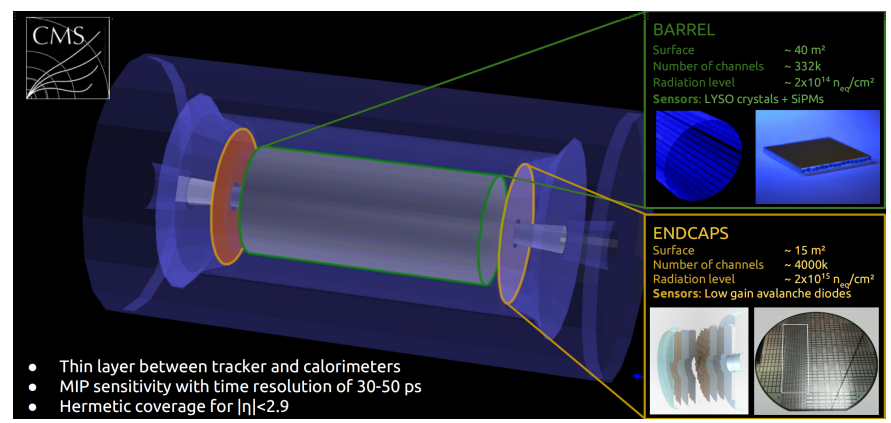

Figure 4: A schematic view of the geometry, implemented in the Geant4 simulation, of the timing layers comprising a LYSO barrel (grey cylinder), at the interface between the tracker and the ECAL, and two silicon endcaps (orange and light violet discs) timing layers in front of the endcap calorimeter.

have been chosen to instrument these two detector regions. Additional constraints from integration within the existing CMS detector, cost and power budgets led to the choice of LYSO:Ce scintillating crystal bars readout with SiPMs for the barrel and Low Gain Avalanche Detectors (LGAD) for the endcaps.

Density of tracks is also different in the barrel and endcap regions, defining different sizes of the active elements to maintain their occupancy below a few percent. This requirement ensures that useful timing information is available for as many charged tracks as possible and yields a large probability for single hits, needed for unambiguous time assignment. A maximum perchannel area of order $1-2 \mathrm{~cm}^{2}$ is suitable for the BTL while for the ETL, a sensor cell-size of $\sim 2 \mathrm{~mm}^{2}$ at $|\eta| \sim 3$ is required. In both cases, the choice of the size of an individual detector element is a compromise between occupancy, sensor characteristics, including electronics considerations such as input capacitance and manufacturing issues, total power needs (number of channels), and cost.

The barrel timing layer (BTL). The barrel timing layer consists of a cylindrical detector of $5.2 \mathrm{~m}$ length, an inner radius of $1148 \mathrm{~mm}$ and a maximum radial extent of $40 \mathrm{~mm}$, located between the outer tracker and the calorimeter. As active elements it exploits arrays of LYSO:Ce crystal bars wrapped with reflective material and readout at both ends with Silicon Photomultipliers as shown in Fig. 5. A total number of 331776 SiPMs (readout channels) with active area of about $3 \times 3 \mathrm{~mm}^{2}$ is used to detect the light from each end of the $3 \times 3 \times 56 \mathrm{~mm}^{3}$ crystals. Scintillating crystals readout with SiPMs have been demonstrated to achieve excellent time resolution [3, 4]. The choice of an elongated geometry for the scintillator allows to reduce the channel count with small loss in performance since light is guided by total internal reflection to the photodetector with small attenuation. Among several scintillators considered, LYSO:Ce was found to be the optimal choice in terms of performance (high light yield and fast scintillation kinetics), cost and mass production capability. It also meets the radiation tolerance requirements as demonstrated in Ref. [5, 6, 7]. The impact of radiation damage on the SiPM performance has been widely studied in Ref. [8, 9]. SiPMs have been proven capable to withstand the integrated radiation levels foreseen for 

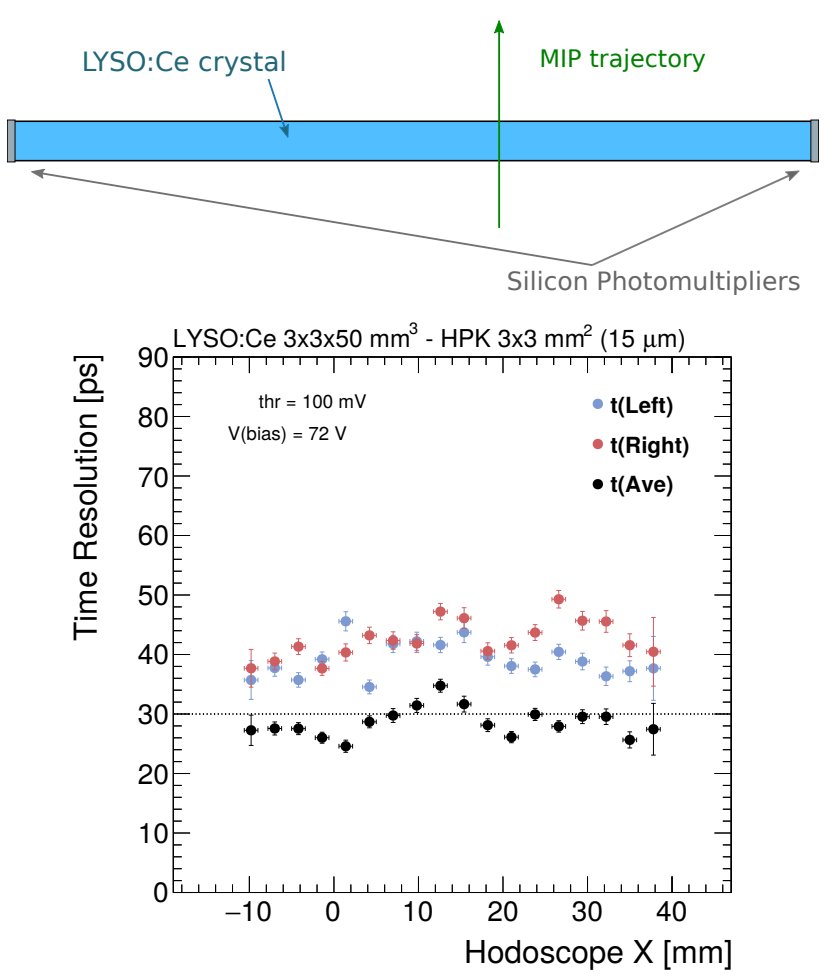

Figure 5: Top: sketch of BTL sensor consisting of a crystal bar with double ended SiPM readout. Bottom: time resolution of a BTL sensor measured in test beam as a function of the MIP impact point across a $50 \mathrm{~mm}$ long crystal bar, by using individual left (blue) and right (red) SiPM and by averaging their time stamp (black).

the barrel region at the end of the detector operation of about $2 \times 10^{14} 1 \mathrm{MeV}$ neq/ $/ \mathrm{cm}^{2}$. The cell pitch of the SiPM which maximizes the photon detection efficiency over the radiation induced dark count noise (DCR), was found to be $15 \mu \mathrm{m}$. The use of small cell pitch devices also allows to reduce the power consumption and extend the dynamic range. The SiPM are readout using a dedicated ASIC, named the TOFHIR (Time-of-flight, High Rate), which provides both amplitude and time measurements with sufficient precision for a group of 32 channels. In order to achieve high precision, the input to the discriminator has to have a very fast rise time, $\mathrm{dV} / \mathrm{dt}$, which requires a substantial amplification and consequently a non-negligible power consumption. The leading edge discriminator is followed by a time-to-digital converter (TDC) with 20 ps binning.

A test beam campaign has proven that $25-30$ ps time resolution can be achieved with LYSO:Ce crystal bars coupled to SiPMs as reported in Fig. 5. The increase of DCR due to radiation during the detector lifetime will contribute with an additional jitter to the time resolution reaching up to $50 \mathrm{ps}$ at the end of detector operation, corresponding to an integrated luminosity of $4000 \mathrm{fb}^{-1}$.

The endcap timing layer (ETL). The endcap timing layer will be instrumented on each end of the barrel, with a hermetic, two-disk system of MIP-sensitive silicon devices providing two hits per track with excellent time resolution. The inner radius and outer radius of each endcap are respectively $315 \mathrm{~mm}$ and

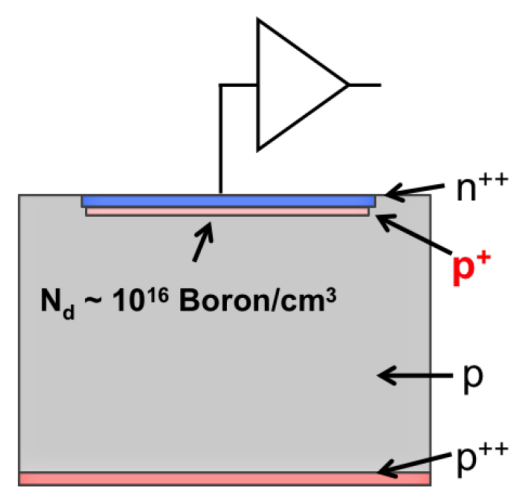

\section{Low gain avalanche detectors}

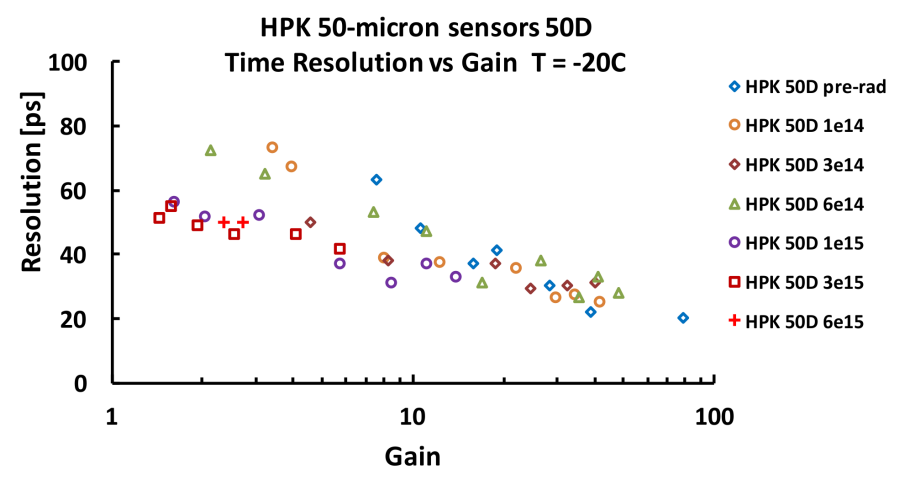

Figure 6: Top: schematic representation of a low gain avalanche detector optimized for timing application by mean of the additional $\mathrm{p}+$ layer underneath the $\mathrm{n}++$ electrode which creates, when depleted, a large electric field that generates charge multiplications. Bottom: time resolution of ETL sensors as a function of gain for different levels of radiation exposure.

$1200 \mathrm{~mm}$ while its total thickness is about $45 \mathrm{~mm}$. The active elements of the ETL consist of Low Gain Avalanche Detectors (LGAD) which are silicon detectors with intrinsic gain of 10-30 provided by a special implant, as shown in Fig. 6, designed to overcome capacitance and other noise sources. Such design provides a low-jitter fast-rising pulse edge that enables precision timing reconstruction for MIPs [10, 11]. The LGAD sensors feature a better radiation tolerance than SiPMs and are thus suitable for instrumentation of the endcap [12, 13]. Sensors with a thin implanted gain layer of overall thickness of 35-50 $\mu \mathrm{m}$ are expected to achieve the desired time resolution.

As shown in Fig. 6, radiation tolerance and beam test studies demonstrated a resolution of about 30 and $50 \mathrm{ps}$ at fluences corresponding to $|\eta| \simeq 2.5$ and 3.0, respectively, at the end of the HL-LHC operation. Achieving good time performance at low-gain requires cell sizes typically less than $2 \mathrm{~mm}^{2}$, to limit the sensor capacitance. Such small pads are grouped in $16 \times 32$ arrays to form a module, of overall dimensions $21 \times 42 \mathrm{~mm}^{2}$, readout by two ASICs, called ETROCs (Endcap Timing Readout Chips). The readout chip uses timing of the leading edge of the pulse from the LGADs and contains amplifiers and discriminators followed by circuits to measure the Time-of-Arrival of each particle and Time-over-Threshold to measure the pulse height for time walk correction. 


\section{Summary}

The development of an innovative detector, the CMS MTD, with capability of tagging charged particles originating from HL-LHC collisions with a time resolution of $30-50$ ps has been presented. The MTD features two different types of sensors: LYSO:Ce crystals readout with SiPMs and silicon sensors with internal gain (LGADs), representing the optimal choice to instrument respectively the barrel and the endcap regions of the CMS detector within integration, cost and power constraints. The accurate time measurement provided by the MTD will bring additional time-of-flight capabilities to CMS, enhancing its performance in reconstructing physics objects under the high pileup conditions characteristic of the HL-LHC operation. This additional handle in mitigating pileup effects yields significant improvements to many physics analyses - including Higgs, LLPs, and di-Higgs physics - by increasing signal efficiencies and improving background rejection.

\section{References}

[1] Technical Proposal for a MIP Timing Detector in the CMS experiment Phase 2 upgrade Tech. Rep. CERN-LHCC-2017-027. LHCC-P-009, CERN, Geneva (Dec 2017).

URL https://cds . cern. ch/record/2296612

[2] Technical Proposal for the Phase-II Upgrade of the CMS Detector Tech. Rep. CERN-LHCC-2015-010. LHCC-P-008, CERN, Geneva (Jun 2015). URL https://cds . cern. ch/record/2020886

[3] A. Benaglia, S. Gundacker, P. Lecoq, M. T. Lucchini, A. Para, K. Pauwels, E. Auffray, Detection of high energy muons with sub-20 ps timing resolution using L(Y)SO crystals and SiPM readout, Nucl. Instrum. Meth. A 830 (2016) 30. doi:10.1016/j.nima.2016.05.030

[4] M. Lucchini, S. Gundacker, P. Lecoq, A. Benaglia, M. Nikl, K. Kamada, A. Yoshikawa, E. Auffray, Timing capabilities of garnet crystals for detection of high energy charged particles Nucl. Instrum. Meth. A 852 (2017) 1. doi:https://doi.org/10.1016/j.nima.2017.02.008 URL http://www.sciencedirect.com/science/article/pii/ S0168900217301900

[5] E. Auffray, A. Barysevich, A. Fedorov, M. Korjik, M. Koschan, M. Lucchini, V. Mechinski, C. Melcher, A. Voitovich, Radiation damage of LSO crystals under $\gamma$ and $24 \mathrm{GeV}$ protons irradiation Nucl.Instrum. Meth. A 721 (2013) 76 . doi:https: //doi.org/10.1016/j.nima.2013.04.065

URL http://www.sciencedirect.com/science/article/pii/ S0168900213004865

[6] F. Yang, et al., Proton induced radiation damage in fast crystal scintillators Nucl. Instrum. Meth. A (2016) 726doi: 10.1016/j.nima.2015.11.100

URL http://www.sciencedirect.com/science/article/pii/ S0168900215014746

[7] F. Yang, L. Zhang, R. Y. Zhu, Gamma-ray induced radiation damage up to $340 \mathrm{Mrad}$ in various scintillation crystals, IEEE Trans. Nucl. Sci. 63 (2) (2016) 612-619. doi:10.1109/TNS.2015.2505721

[8] E. Garutti, Y. Musienko, Radiation damage of SiPMsarXiv:1809. 06361

[9] A. Heering, Y. Musienko, R. Ruchti, M. Wayne, A. Karneyeu, V. Postoev, Effects of very high radiation on SiPMs Nucl. Instrum. Meth. A 824 (2016) 111. doi : 10.1016/j.nima.2015.11.037

URL http://www.sciencedirect.com/science/article/pii/ S0168900215014023

[10] G. Pellegrini, et al., Technology developments and first measurements of Low Gain Avalanche Detectors (LGAD) for high energy physics applications Nucl. Instrum. Meth. A 765 (2014) 12. doi:10.1016/j.nima.2014.06.008

URL http://www.sciencedirect.com/science/article/pii/ S0168900214007128
[11] N. Cartiglia, et al., Design optimization of ultra-fast silicon detectors, Nucl. Instrum. Meth. A 796 (2015) 141. doi:10.1016/j.nima.2015. 04.025

[12] G. Kramberger, et al., Radiation hardness of thin low gain avalanche detectors Nuclear Instruments and Methods in Physics Research Section A 891 (2018) $68-77$.

URL http://www.sciencedirect.com/science/article/pii/ S0168900218301682

[13] M. Ferrero, R. Arcidiacono, M. Barozzi, M. Boscardin, N. Cartiglia, G. D. Betta, Z. Galloway, M. Mandurrino, S. Mazza, G. Paternoster, F. Ficorella, L. Pancheri, H.-F. W. Sadrozinski, F. Siviero, V. Sola, A. Staiano, A. Seiden, M. Tornago, Y. Zhao, Radiation resistant lgad design Nuclear Instruments and Methods in Physics Research Section A: Accelerators, Spectrometers, Detectors and Associated Equipment 919 (2019) 16 - 26. doi:https://doi.org/10.1016/j.nima.2018.11.121 URL http://www.sciencedirect.com/science/article/pii/ S0168900218317741 